\title{
DISCOVERY OF A VERY HIGHLY EXTINGUISHED SUPERNOVA IN A LUMINOUS INFRARED GALAXY
}

\author{
E. Kankare, ${ }^{1}$ S. Mattila, ${ }^{1}$ S. Ryder, ${ }^{2}$ M.-A. Pérez-Torres,${ }^{3}$ A. Alberdi,${ }^{3}$ C. Romero-Canizales, ${ }^{3}$ T. Díaz-Santos, ${ }^{4}$ \\ P. Väisänen, ${ }^{5}$ A. Efstathiou, ${ }^{6}$ A. Alonso-Herrero, ${ }^{4}$ L. Colina, ${ }^{4}$ and J. Kotilainen ${ }^{1}$ \\ Received 2008 September 2; accepted 2008 October 22; published 2008 November 7
}

\begin{abstract}
We report the discovery of a confirmed supernova $(\mathrm{SN})$ and a supernova candidate in near-infrared images from the ALTAIR/NIRI adaptive optics system on the Gemini-North Telescope and NICMOS on the Hubble Space Telescope. The Gemini images were obtained as part of a near-infrared $K$-band search for highly obscured $\mathrm{SNe}$ in the nuclear regions of luminous infrared galaxies. SN 2008cs, apparent in the Gemini images, is the first SN discovered using laser guide star adaptive optics. It is located at $1500 \mathrm{pc}$ projected distance from the nucleus of the luminous infrared galaxy IRAS 17138-1017. The SN luminosity, JHK colors, and light curve are consistent with a core-collapse event suffering from a very high host galaxy extinction of $15.7 \pm 0.8 \mathrm{mag}$ in the $V$-band, which is to our knowledge the highest yet measured for a SN. The core-collapse nature of SN 2008cs is confirmed by its radio detection at $22.4 \mathrm{GHz}$ using our Very Large Array observations 28 days after the SN discovery, indicating a prominent interaction of the SN ejecta with the circumstellar medium. An unconfirmed SN apparent in the NICMOS images from 2004 is located in the same galaxy at $660 \mathrm{pc}$ projected distance from the nucleus and has a lower extinction.
\end{abstract}

Subject headings: galaxies: individual (IRAS 17138-1017) — galaxies: starburst — infrared: galaxies instrumentation: adaptive optics — supernovae: individual (SN 2008cs)

Online material: color figures

\section{INTRODUCTION}

A large fraction of the massive star formation at high $z$, e.g., $70 \%$ at $z=1$ (Le Floc'h et al. 2005), took place in luminous $\left(L_{\mathrm{IR}}>10^{11} L_{\odot}\right)$ and ultraluminous $\left(L_{\mathrm{IR}}>10^{12} L_{\odot}\right)$ infrared (IR) galaxies (LIRGs and ULIRGs, respectively), and their high star formation rates (SFRs) can be expected to result in core-collapse supernova (CCSN) rates a couple of orders of magnitude higher than in ordinary field galaxies. Most of the SNe occurring in LIRGs and ULIRGs are likely to be obscured by large amounts of dust in the nuclear starburst environment; however, in the near-IR the extinction is strongly reduced. Because of the likely concentration of most of the SNe within the innermost nuclear regions, a high spatial resolution is crucial for their detection. This can be achieved with space-based imaging (e.g., Cresci et al. 2007; Colina et al. 2007), imaging from Antarctica (see Burton et al. 2005), or ground-based adaptive optics (AO) imaging providing resolutions 5 times better than under typical natural seeing conditions. The potential of the current $8 \mathrm{~m}$ class telescopes equipped with $\mathrm{AO}$ was recently demonstrated by the discovery of SN 2004ip in the LIRG nuclear regions using the NACO AO system with a natural guide star on the Very Large Telescope (Mattila et al. 2007).

In this Letter we report the discovery of two SNe in IRAS 17138-1017, a LIRG $\left(L_{\mathrm{IR}}=2.4 \times 10^{11} L_{\odot}\right.$; Sanders et al. 2003) at redshift $z=0.0173$ (Shier \& Fischer 1998), corresponding to a distance of $75 \mathrm{Mpc}\left(H_{0}=70 \mathrm{~km} \mathrm{~s}^{-1} \mathrm{Mpc}^{-1}\right.$, $\Omega_{\Lambda}=0.7$, and $\Omega_{M}=0.3$ ). The SNe were detected as a result

\footnotetext{
${ }^{1}$ Tuorla Observatory, Department of Physics and Astronomy, University of Turku, Väisäläntie 20, FI-21500 Piikkiö, Finland; eskank@utu.fi.

${ }^{2}$ Anglo-Australian Observatory, PO Box 296, Epping, NSW 1710, Australia.

${ }^{3}$ Instituto de Astrofísica de Andalucia, IAA-CSIC, Apartado 3004, 18080 Granada, Spain.

${ }^{4}$ Instituto de Estructura de la Materia IEM-CSIC, 28006 E-Madrid, Spain.

${ }^{5}$ South African Astronomical Observatory, PO Box 9, Observatory 7935, South Africa.

${ }^{6}$ School of Sciences, European University Cyprus, Diogenes Street, Engomi, 1516 Nicosia, Cyprus.
}

of our $K$-band search for highly obscured CCSNe in a sample of nearby LIRGs using the Gemini-North Telescope. Even though IRAS $17138-1017$ has a high expected SN rate, only one SN (the Type Ia SN 2002bw, with a likely low extinction; Li 2002; Matheson et al. 2002) has been detected in this galaxy before.

\section{OBSERVATIONS AND RESULTS}

\subsection{IR Observations}

IRAS $17138-1017$ was observed with HST NICMOS as part of a program to observe a complete sample of LIRGs (Alonso-Herrero et al. 2006) with the NIC2 camera (0.076" pixel $^{-1}$, FWHM $\sim 0.1^{\prime \prime}-0.2^{\prime \prime}$ ) on 2004 September 23.0 UT (see Table 1). Observations were conducted with the F110W and F160W broadband filters and with the F187N narrowband filter. Details of the data reduction and photometric calibration of the NICMOS images can be found in Alonso-Herrero et al. (2006). The LIRG was next observed with the Gemini-North Telescope $^{7}$ in the $K$-band using the Near-InfraRed Imager (NIRI) with the ALTAIR laser guide star AO system (0.022" pixel $^{-1}$, FWHM 0.1") on 2008 April 21.6 UT (program GN-2008AQ-38, PI: S. Ryder). The NIRI images were reduced using the NIRI package in IRAF. The target frames were dedithered and median combined in IRAF using a bright field star as a reference point. Comparison between the HST and Gemini observations revealed two new point sources, one at each epoch. The object apparent only in the HST images is located at $1.9^{\prime \prime}$ (or $660 \mathrm{pc}$ ), and the one seen only in the Gemini images at $4.2^{\prime \prime}$ (or $1500 \mathrm{pc}$ ) projected distance from the near-IR nucleus

\footnotetext{
${ }^{7}$ Based on observations obtained at the Gemini Observatory, which is operated by the Association of Universities for Research in Astronomy, Inc., under a cooperative agreement with the NSF on behalf of the Gemini partnership: the National Science Foundation (US), the Science and Technology Facilities Council (UK), the National Research Council (Canada), CONICYT (Chile), the Australian Research Council (Australia), Ministario da Ciancia e Tecnologia (Brazil), and SECYT (Argentina).
} 
TABLE 1

Log of the Observations and Supernova Photometry

\begin{tabular}{|c|c|c|c|}
\hline UT Date & Instrument, Filter & $\begin{array}{l}\text { Exposure } \\
\text { Time } \\
\text { (s) }\end{array}$ & Magnitude \\
\hline \multicolumn{4}{|c|}{ Possible $2004 \mathrm{SN}$} \\
\hline \multirow[t]{2}{*}{2004 Sep $23.0 \ldots \ldots .}$. & NICMOS, F110W & $3 \times 80$ & $18.28 \pm 0.06$ \\
\hline & NICMOS, F160W & $3 \times 80$ & $17.00 \pm 0.05$ \\
\hline \multirow[t]{2}{*}{2004 Sep $23.1 \ldots \ldots}$. & NICMOS, F187N & $3 \times 288$ & $16.58 \pm 0.06$ \\
\hline & SN $2008 \mathrm{cs}$ & & \\
\hline \multirow{4}{*}{$\begin{array}{l}2008 \text { Apr } 21.6 \ldots \ldots \\
2008 \text { May } 20.5 \ldots \ldots\end{array}$} & ALTAIR/NIRI, $K$ & $8 \times 30$ & $16.65 \pm 0.06$ \\
\hline & NIRI, $K$ & $9 \times 30$ & $16.22 \pm 0.37$ \\
\hline & NIRI, $H$ & $7 \times 30$ & $17.40 \pm 0.46$ \\
\hline & NIRI, $J$ & $9 \times 30$ & $19.43 \pm 0.50$ \\
\hline \multirow[t]{2}{*}{2008 May $27.5 \ldots \ldots$} & ALTAIR/NIRI, $K$ & $8 \times 30$ & $15.77 \pm 0.06$ \\
\hline & ALTAIR/NIRI, $H$ & $8 \times 30$ & $16.97 \pm 0.06$ \\
\hline \multirow[t]{3}{*}{2008 Jun $18.1 \ldots \ldots$} & NOTCam, $K_{s}$ & $18 \times 60$ & $15.99 \pm 0.61$ \\
\hline & NOTCam, $H$ & $18 \times 60$ & $16.92 \pm 0.35$ \\
\hline & NOTCam, $J$ & $18 \times 60$ & $19.58 \pm 0.65$ \\
\hline \multirow[t]{3}{*}{2008 Jun 25.5} & ALTAIR/NIRI, $K$ & $8 \times 30$ & $15.82 \pm 0.10$ \\
\hline & ALTAIR/NIRI, $H$ & $8 \times 30$ & $17.17 \pm 0.05$ \\
\hline & ALTAIR/NIRI, $J$ & $8 \times 30$ & $19.02 \pm 0.14$ \\
\hline \multirow[t]{2}{*}{2008 Sep 20.2} & ALTAIR/NIRI, $K$ & $8 \times 30$ & $17.30 \pm 0.09$ \\
\hline & ALTAIR/NIRI, $H$ & $8 \times 30$ & $19.11 \pm 0.09$ \\
\hline 2008 Sep 21.2 & ALTAIR/NIRI, $J$ & $8 \times 30$ & $>21.4$ \\
\hline
\end{tabular}

of IRAS 17138-1017. Both objects were reported to the Central Bureau for Astronomical Telegrams (CBAT) immediately after discovery, and the more recent one was later assigned the designation SN 2008cs (Kankare et al. 2008; Pérez-Torres et al. 2008). Follow-up observations of SN 2008cs were obtained on May 20.5 UT with NIRI in the $J, H$, and $K$ bands without AO $\left(0.117^{\prime \prime}\right.$ pixel $^{-1}$, FWHM $\left.\sim 0.5^{\prime \prime}\right)$, and with ALTAIR/NIRI on May 27.5, June 25.5, and September 20.2-21.2 UT (see Fig. 1) using the AO. Follow-up observations were also obtained using the Nordic Optical Telescope (NOT) with the NOTCam instrument $\left(0.234^{\prime \prime}\right.$ pixel $^{-1}$, FWHM $\left.\sim 0.7^{\prime \prime}\right)$ in the $J$, $H$, and $K_{s}$ bands on June 18.1 UT (see Table 1).

We used 35 stars from the 2MASS catalog to derive an accurate World Coordinate System (WCS) for an HST ACS Iband image of IRAS 17138-1017 obtained from the HST Science Archive. The Gemini/NIRI $K$-band (AO) image from June 25 was aligned to the ACS image using seven pointlike sources common between the frames and a simple geometric transfor- mation including shifts in $x$ and $y$, and the same scale factor and rotation for both $x$ and $y$. This yielded $17^{\mathrm{h}} 16^{\mathrm{m}} 35.855^{\mathrm{s}}$ and $-10^{\circ} 20^{\prime} 42.98^{\prime \prime}$ for SN $2008 \mathrm{cs}$, with $0.09^{\prime \prime}$ and $0.07^{\prime \prime}$ uncertainty in R.A. and decl., respectively. The HST NICMOS F160W image was aligned to the ACS image using six point sources, and this yielded R.A. $=17^{\mathrm{h}} 16^{\mathrm{m}} 35.900^{\mathrm{s}}$ and decl. $=$ $-10^{\circ} 20^{\prime} 37.85^{\prime \prime}$ for the 2004 event.

\subsection{SN 2008cs Photometry and Extinction}

The photometry of SN 2008cs was measured from the images using the SNOOPY ${ }^{8}$ package in IRAF. Suitable field stars were selected to fit a point-spread function (PSF) for each image. A polynomial surface was fitted to a background region around the object position (excluding the innermost region around the object), and after removing the local background the PSF was fitted to the object, and a residual image was produced after subtracting the PSF. Error estimates for the measurements were obtained by simulating and PSF fitting nine artificial point sources around the SN in the residual images. For the non-AO Gemini/NIRI and NOT images the photometry was calibrated using six 2MASS stars within the image field of view (FOV). The AO Gemini/NIRI images with small FOV were photometrically calibrated to the non-AO images using PSF photometry of three field stars available in both the AO and non-AO images (see Fig. 1). Assuming that these field stars have a similar AO PSF as the $\mathrm{SN}$, this procedure also corrects for the flux lost in the PSF halo around the diffractionlimited core in the AO images. Finally, all the magnitudes were aperture corrected using three bright, isolated stars in the non$\mathrm{AO}$ images to an aperture of $3^{\prime \prime}$ in radius. For the complete list of apparent SN magnitudes see Table 1.

Assuming a distance of $75 \mathrm{Mpc}$ and a Galactic extinction of $A_{V}=2.29$ (Schlegel et al. 1998, but see also Arce \& Goodman 1999), the absolute magnitude of the $\mathrm{SN}$ at the time of the second AO observation (when the $\mathrm{SN}$ is closest to the peak) becomes $M_{H}=-17.81$ and $M_{K}=-18.87$ (before correcting for the host galaxy extinction). We compared these with the absolute peak magnitudes of typical Type II-P SNe 1999em

${ }^{8}$ SNOOPY, originally presented in Patat (1996), has been implemented in IRAF by E. Cappellaro. The package is based on DAOPHOT, but optimized for SN magnitude measurements.

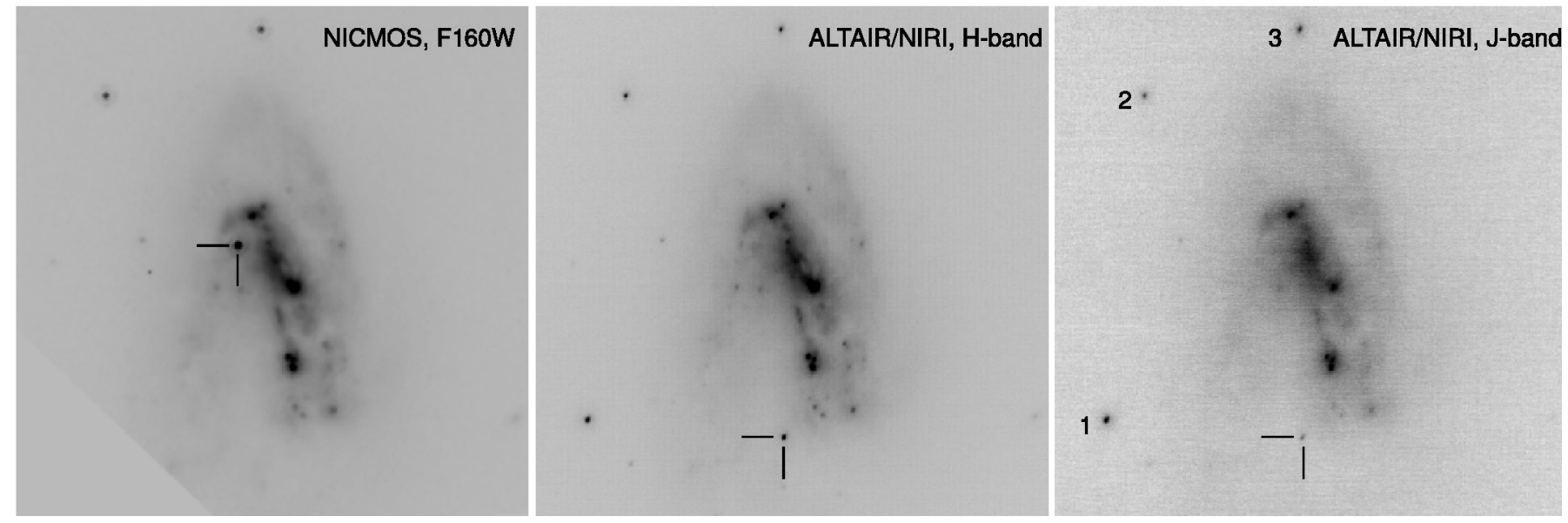

FIG. 1.-14" $\times 14^{\prime \prime}$ subsections of IRAS 17138-1017 images taken with the HST NICMOS F160W filter on 2004 September 23, and with Gemini ALTAIR/ NIRI AO in the $H$ and $J$ bands on 2008 June 25. The "historical" SN from 2004 and SN 2008cs are indicated in the NICMOS and Gemini images, respectively. The effects of high extinction in the SN 2008cs line of sight can also be seen by comparing the brightness of the SN to the field stars in the $H$ and $J$ bands. The three field stars indicated in the right-hand panel were used for photometric calibration. North is up and east is to the left in all the images. 


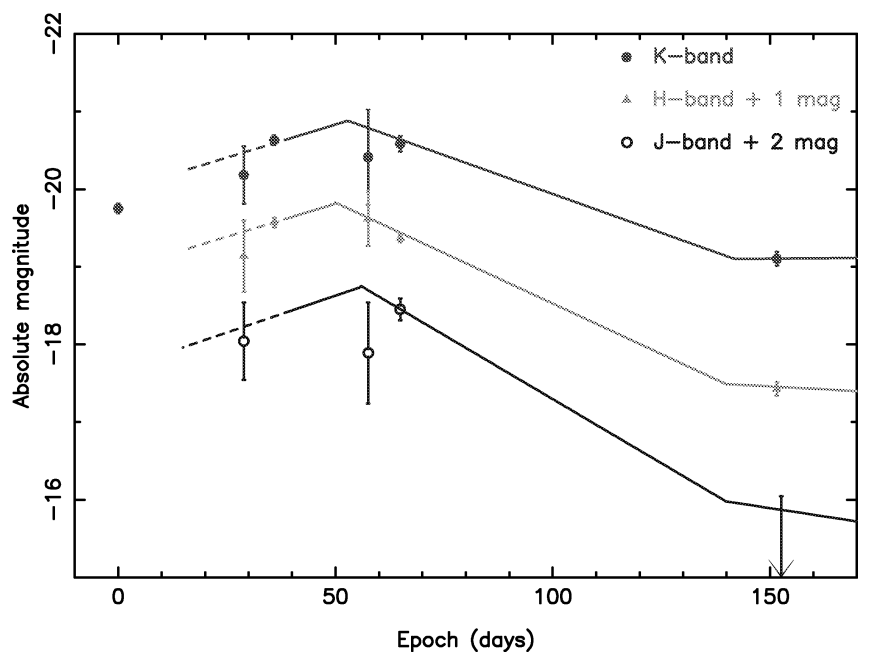

FIG. 2.-Example fit for the SN 2008cs light curves with $A_{V}$ (host) $=15.8$. The SN is 0.9 mag brighter than the "slowly declining" CCSN template light curves, and the $K$-band peak occurred at 53 days after the discovery; see text for details. [See the electronic edition of the Journal for a color version of this figure.]

and 2003hn $\left(M_{H} \approx-17.8\right.$ and $M_{K} \approx-18.1$; Krisciunas et al. $2008)$ and the "ordinary" $\left(M_{H}=-18.58\right.$ and $\left.M_{K}=-18.62\right)$ and "slowly declining" $\left(M_{H}=-19.95\right.$ and $\left.M_{K}=-20.02\right)$ CCSN template light curves from Mattila \& Meikle (2001). The observed SN color and peak absolute magnitude can only be accounted for by the "slowly declining" type, and even then only with a substantial host galaxy extinction. Adopting these $J H K$ templates and the extinction law of Rieke \& Lebofsky (1985), for which $A_{K}=0.112 A_{V}$, we obtain a host galaxy extinction toward SN 2008cs of $1.67-1.85 \mathrm{mag}$ in $K$. This corresponds to $14.9-16.5 \mathrm{mag}$ in $V\left(A_{V}=17.2-18.8\right.$ total lineof-sight extinction), with the intrinsic absolute peak magnitude $M_{K}=-20.9 \pm 0.1$ (statistical error only) occurring around 49-55 days after the discovery (see Fig. 2 for an example fit). We note that in NICMOS images there is no star-forming region detected at the position of SN 2008cs, as expected due to the high extinction toward the SN (see Díaz-Santos et al. 2008).

\subsection{The CCSN Rate of IRAS 17138-1017}

To estimate the SFR for IRAS 17138-1017, we compiled its $12-100 \mu \mathrm{m} I R A S$ photometry and fitted the resulting spectral energy distribution (SED; see Fig. 3) with the starburst models of Efstathiou et al. (2000). The starburst model provides a very good fit to the near- to far-IR SED and is consistent with a negligible contribution from an active galactic nucleus (AGN; see also DePoy et al. 1988). The model assumes an exponentially decaying starburst with a time constant of $30 \mathrm{Myr}$ and an age of $57 \mathrm{Myr}$. The initial $A_{V}$ of the molecular clouds that constitute the starburst is $100 \mathrm{mag}$. The SFR at the peak is 169 $M_{\odot} \mathrm{yr}^{-1}$, and $76 M_{\odot} \mathrm{yr}^{-1}$ when averaged over the duration of the starburst. We estimate a bolometric luminosity of $2.3 \times$ $10^{11} L_{\odot}$ for IRAS $17138-1017$, which is close to its IR luminosity, as expected for a LIRG. Adopting the average SFR and assuming CCSN progenitors between 8 and $50 M_{\odot}$ and a Salpeter initial mass function with cutoffs at 0.1 and $125 M_{\odot}$, we obtain a CCSN rate of $\sim 0.53 \mathrm{yr}^{-1}$ for IRAS $17138-1017$. Using $76 M_{\odot} \mathrm{yr}^{-1}$ as an upper limit for the SFR averaged over $0.5 \mathrm{Gyr}$, we obtain an upper limit for its Type Ia SN rate (Sullivan et al. 2006) of $\sim 0.03 \mathrm{yr}^{-1}$, which is $\sim 20$ times smaller than the estimated CCSN rate.

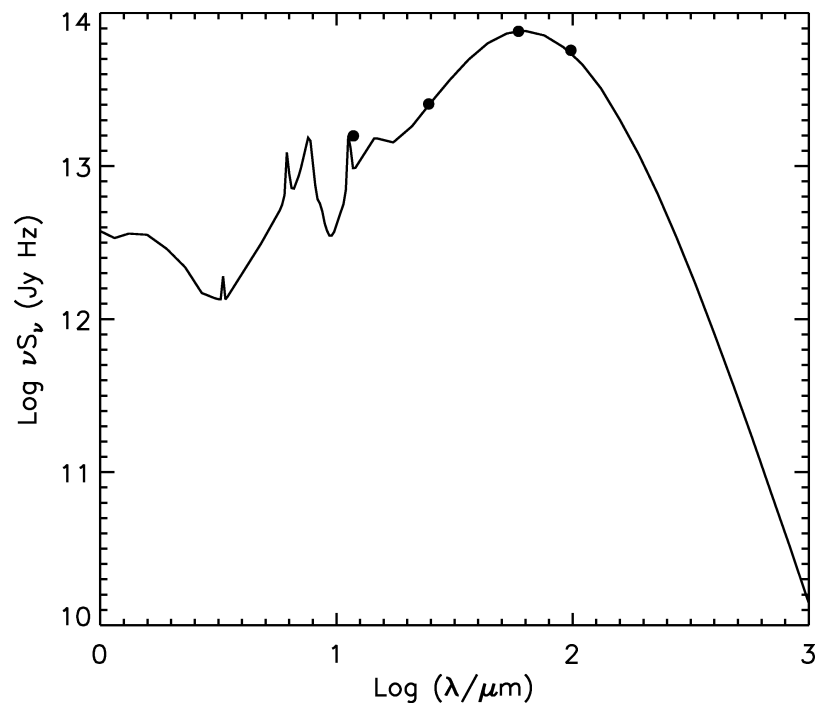

FIG. 3.-IR SED of IRAS 17138-1017 fitted with the starburst models of Efstathiou et al. (2000). No AGN contribution is needed to explain the SED.

\subsection{VLA Observations}

We observed IRAS 17138-1017 on 2008 May 19.4 UT (program AP558, PI: M. Pérez-Torres) at 8.4 and $22.4 \mathrm{GHz}$, using the Very Large Array (VLA) in the C configuration, aimed at detecting any radio emission from $\mathrm{SN} 2008 \mathrm{cs}$ with rapid response exploratory VLA time. The observations lasted for $2.5 \mathrm{hr}$, with effective on-source times on the nucleus of IRAS 17138-1017 of $\sim 60$ and $\sim 49$ minutes at 8.4 and $22.4 \mathrm{GHz}$, respectively. We used the source 1713-130 for phase and amplitude calibration purposes, and the quasar 3C $286(1331+305)$ to set the absolute flux density scale. We then edited, calibrated, and imaged our 8.4 and $22.4 \mathrm{GHz}$ VLA data, following standard data reduction techniques implemented within the NRAO Astronomical Image Processing System (AIPS). Since the VLA was in C configuration, the angular resolution of the $8.4 \mathrm{GHz}$ observations did not allow the detection of any compact radio emission, apart from that of the nuclear region. But our $22.4 \mathrm{GHz}$ (beam size $\sim 0.9^{\prime \prime}$ ) observations clearly showed a local maximum of radio emission $\left(S_{v}=440 \pm 75 \mu \mathrm{Jy}\right.$; Pérez-Torres et al. 2008) at R.A. $=17^{\mathrm{h}} 16^{\mathrm{m}} 35.853^{\mathrm{s}}$, decl. $=-10^{\circ} 20^{\prime} 43.05^{\prime \prime}$. The radio source appears coincident within the uncertainties $\left(0.2^{\prime \prime}\right.$ in each coordinate) with the position of the SN at near-IR wavelengths (offset of $0.03^{\prime \prime}$ and $0.07^{\prime \prime}$ in R.A. and decl., respectively), which confirms that both the near-IR and radio emission come from the same region (see Fig. 4). Future higher angular resolution observations should also detect SN 2008cs at longer wavelengths.

Type Ia $\mathrm{SNe}$ are not expected to be strong radio emitters and have not been detected at radio wavelengths (e.g., Panagia et al. 2006). Our detection of SN 2008cs at $22.4 \mathrm{GHz}$ indicates a prominent interaction of the SN ejecta with the circumstellar medium, confirming that the SN was a core-collapse event. SN $2008 \mathrm{cs}$ is only the third SN, after SNe 2000ft (Colina et al. 2001) and 2004ip (Pérez-Torres et al. 2007), detected at both optical/IR and radio wavelengths in a LIRG/ULIRG host.

\subsection{The "Historical" Supernova from 2004}

We carried out the photometry of the 2004 event with circular apertures and subtracted the local background. The aperture corrections were done using synthetic PSFs generated with TinyTim (Krist et al. 1998). An asteroid origin for the 2004 source was immediately ruled out, since it is stationary compared to the field 


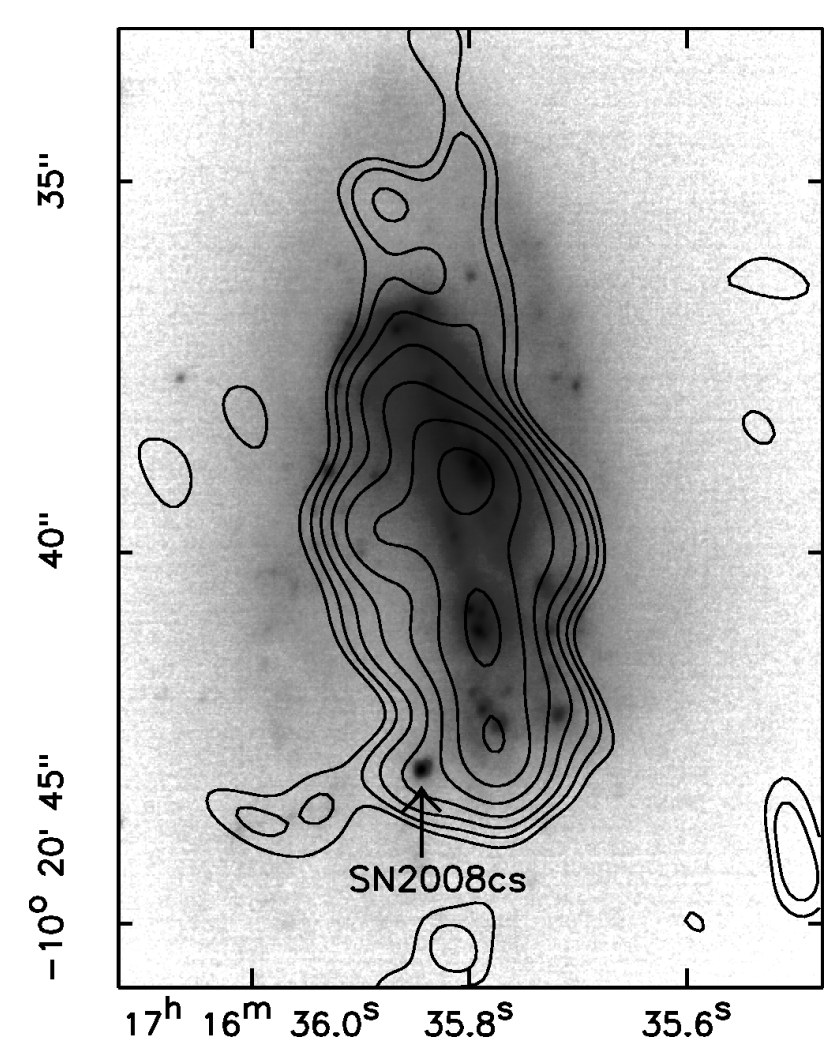

FIG. 4.- $K$-band Gemini ALTAIR/NIRI image from 2008 June 25 with 22.4 GHz VLA-C contours from 2008 May 19 overlaid. [See the electronic edition of the Journal for a color version of this figure.]

stars between the first and the last target frame, separated by $\sim 40$ minutes. Also, no known minor planets were near the source within a radius of $10^{\prime}$ at the time of the observation. A variable foreground star also provides a very improbable alternative explanation given the small FOV $\left(19.8^{\prime \prime} \times 19.8^{\prime \prime}\right)$ of the HST NICMOS frames. For example, the likelihood of catching a foreground nova would be extremely small given the Galactic nova rate of $\sim 35 \mathrm{yr}^{-1}$ (Shafter 1997). Based on this and the high CCSN rate of IRAS 17138-1017, we conclude that the 2004 transient source was very likely a CCSN. From its $(J-H)$ color and assuming a negligible difference between the F160W and the ground-based $H$ filter and a conversion from the F110W to the ground-based $J$-band photometry (Origlia \& Leitherer 2000), as well as the template CCSN light curves from Mattila \& Meikle (2001), we can estimate a host galaxy extinction for the SN. We obtain $A_{V} \approx 4$ mag if it was an "ordinary" CCSN near the peak, and $A_{V} \approx 0$ mag if it was a "slowly declining" CCSN at $\sim 4$ months from the peak.

\section{CONCLUSIONS}

Near-IR-discovered SNe in LIRGs and ULIRGs are not only important for SN rate estimates. They can also be used to estimate the amount and distribution of extinction within their host galaxies, and follow-up observations can provide us with a better understanding of the behavior of SNe within their dusty, high-density environments. To our knowledge, the host galaxy extinction of $A_{V}=15.7 \pm 0.8$ mag measured for SN $2008 \mathrm{cs}$ is the highest observed for a SN so far. Previous events with high extinctions include SN 2001db $\left(A_{V} \approx 5.5\right.$; Maiolino et al. 2002), SN 2002cv ( $A_{V} \approx 9$; Di Paola et al. 2002; Elias-Rosa et al. 2008), SN 2002hh ( $A_{V} \approx 5$; Pozzo et al. 2006), SN 2004am ( $A_{V} \approx 5$; Mattila et al., in preparation), and SN 2004ip $\left(A_{V} \approx\right.$ 5-40; Mattila et al. 2007). Such high-extinction events can have an important impact for the SN statistics when estimating the complete CCSN rates, including also the optically obscured $\mathrm{SNe}$. This will be essential when using CCSNe as probes of the SFR at both low and high $z$ (e.g., Cappellaro et al. 1999; Dahlen et al. 2004; Botticella et al. 2008; Melinder et al. 2008), with the aim of providing a new independent measurement of the cosmic star formation history.

We thank Peter Meikle for useful comments and Harry Lehto for useful discussions. E. K. acknowledges support from the Finnish Academy of Science and Letters (Vilho, Yrjö, and Kalle Väisälä Foundation). S. M. and J. K. acknowledge support from the Academy of Finland (projects 8120503 and 8121122, respectively). M. A. P-T., A. A., and C. R-C. acknowledge support from the Spanish grant AYA2006-14986-C02-C01. T. D.-S., A. A.-H., and L. C. acknowledge support from the grant ESP200765475-C02-01.

\section{REFERENCES}

Alonso-Herrero, A., Rieke, G. H., Rieke, M. J., Colina, L., Pérez-González, P. G., \& Ryder, S. D. 2006, ApJ, 650, 835

Arce, H. G., \& Goodman, A. A. 1999, ApJ, 512, L135

Botticella, M. T., et al. 2008, A\&A, 479, 49

Burton, M. G., et al. 2005, Publ. Astron. Soc. Australia, 22, 199

Cappellaro, E., Evans, R., \& Turatto, M. 1999, A\&A, 351, 459

Colina, L., Alberdi, A., Torrelles, J. M., Panagia, N., \& Wilson, A. S. 2001, ApJ, 553, L19

Colina, L., Díaz-Santos, T., Alonso-Herrero, A., Panagia, N., Alberdi, A., Torrelles, J. M., \& Wilson, A. S. 2007, A\&A, 467, 559

Cresci, G., Mannucci, F., Della Valle, M., \& Maiolino, R. 2007, A\&A, 462, 927

Dahlen, T., et al. 2004, ApJ, 613, 189

DePoy, D. L., Wynn-Williams, C. G., Hill, G. J., \& Becklin, E. E. 1988, AJ, 95, 398

Di Paola, A., Larionov, V., Arkharov, A., Bernardi, F., Caratti o Garatti, A., Dolci, M., Di Carlo, E., \& Valentini, G. 2002, A\&A, 393, L21

Díaz-Santos, T., Alonso-Herrero, A., Colina, L., Packham, C., Radomski, J. T., \& Telesco, C. M. 2008, ApJ, 685, 211

Efstathiou, A., Rowan-Robinson, M., \& Siebenmorgen, R. 2000, MNRAS, 313,734

Elias-Rosa, N., et al. 2008, MNRAS, 384, 107

Kankare, E., et al. 2008, CBET, 1392, 1

Krisciunas, K., et al. 2008, preprint (arXiv:0809.2591)
Krist, J. E., Golimowski, D. A., Schroeder, D. J., \& Henry, T. J. 1998, PASP, 110,1046

Le Floc'h, E., et al. 2005, ApJ, 632, 169

Li, W. D. 2002, IAU Circ., 7864, 2

Maiolino, R., et al. 2002, A\&A, 389, 84

Matheson, T., Jha, S., Challis, P., Kirshner, R., \& Calkins, M. 2002, IAU Circ., 7872,2

Mattila, S., \& Meikle, W. P. S. 2001, MNRAS, 324, 325

Mattila, S., et al. 2007, ApJ, 659, L9

Melinder, J., Mattila, S., Östlin, G., Mencía Trinchant, L., \& Fransson, C. 2008, A\&A, 490, 419

Origlia, L., \& Leitherer, C. 2000, AJ, 119, 2018

Panagia, N., et al. 2006, ApJ, 646, 369

Patat, F. 1996, Ph.D. thesis, Univ. Padova

Pérez-Torres, M. A., et al. 2007, ApJ, 671, L21 2008, CBET, 1392, 2

Pozzo, M., et al. 2006, MNRAS, 368, 1169

Rieke, G. H., \& Lebofsky, M. J. 1985, ApJ, 288, 618

Sanders, D. B., Mazzarella, J. M., Kim, D.-C., Surace, J. A., \& Soifer, B. T. 2003, AJ, 126, 1607

Schlegel, D. J., Finkbeiner, D. P., \& Davis, M. 1998, ApJ, 500, 525

Shafter, A. W. 1997, ApJ, 487, 226

Shier, L. M., \& Fischer, J. 1998, ApJ, 497, 163

Sullivan, M., et al. 2006, ApJ, 648, 868 